\title{
Pulmonary Infections Following Lung Transplantation
}

Chad A. Witt, MD ${ }^{a, *}$, Bryan F. Meyers, MD, MPH ${ }^{b}$, Ramsey R. Hachem, MDa

\section{KEYWORDS}

- Lung transplantation • Immunocompromised • Infectious complications • Cytomegalovirus

- Aspergillosis • Prophylaxis

\section{KEY POINTS}

- Lung transplant recipients are at a higher risk of infectious complications than recipients of other solid organs.

- The major cause of mortality beyond 1 year after lung transplantation is bronchiolitis obliterans syndrome (chronic allograft rejection).

- Infections with cytomegalovirus (CMV), Pseudomonas aeruginosa, and Aspergillus species have been associated with the development of bronchiolitis obliterans syndrome.

- CMV and fungal prophylaxis regimens vary by transplant center.

- Pneuomcystis jiroveci pneumonia is rare in the era of prophylaxis.

\section{INTRODUCTION}

In 2009, 3272 lung transplant procedures were reported to the International Society for Heart and Lung Transplantation for end-stage lung disease, the highest annual number to date. Survival for lung transplant recipients continues to lag behind that of other solid organ transplant recipients, with a median survival ("half-life") of 5.5 years. ${ }^{1}$ Graft failure and non-cytomegalovirus (non-CMV) infection remain the major reported causes of postoperative mortality in the first year after transplantation. After the first year, the most common identifiable causes for mortality are bronchiolitis obliterans syndrome (BOS) and non-CMV infection. Immunosuppression contributes significantly to posttransplant infections in all recipients; however, lung transplant recipients are generally more intensively immunosuppressed than other solid organ recipients and have more frequent and more severe infectious complications. In addition, factors such as preoperative microorganism colonization, transmission of infectious agents from the donor organs, blunted cough mechanism due to denervation, impaired mucociliary clearance, poor lymphatic drainage, ischemic large airways in the immediate postoperative period, and constant exposure to the environment uniquely place lung transplant recipients at higher risk for infections than other organ recipients. ${ }^{2}$

Chronic allograft rejection, in the form of BOS, is the major life-limiting complication after lung transplantation. Pulmonary infections with viruses, including CMV; gram-negative bacilli, especially Pseudomonas aeruginosa; and Aspergillus species have all been associated with an increased risk of BOS. $^{3-7}$ These findings underscore the importance of prevention, diagnosis, and treatment of infections in the population that has undergone lung transplantation.

\footnotetext{
a Department of Internal Medicine, Washington University School of Medicine in St Louis, 660 South Euclid Avenue Campus Box 8052, St Louis, MO 63110, USA; ${ }^{b}$ Department of Surgery, Washington University School of Medicine in St Louis, 660 South Euclid Avenue Campus Box 8234, St Louis, MO 63110, USA

* Corresponding author.

E-mail address: cwitt@dom.wustl.edu
} 


\section{PERIOPERATIVE PERIOD}

During the perioperative period, lung transplant recipients are at increased risk for bacterial, fungal, and viral infections. In this patient population, gram-negative organisms, including $P$ aeruginosa, and methicillin-resistant Staphylococcus aureus (MRSA) are often encountered. ${ }^{8}$ Infections can result from chronic preoperative recipient colonization, as is common in suppurative conditions such as cystic fibrosis (CF). Other mechanisms include acute perioperative donor colonization or infection and postoperative infection in early recipients via mechanical ventilation or through other means throughout the initial hospital stay.

Donor airway sputum specimens are typically obtained for culture in patients before implantation. The results of Gram stain and culture from the donor sputum are followed up to ensure that the initial antibiotic regimen appropriately covers organisms identified in the donor airway specimens. In transplant recipients colonized with known organisms, perioperative antibiotic prophylaxis is based on the results of prior cultures and sensitivity data. This is especially important in patients with CF or non-CF bronchiectasis because these patients are often chronically infected with multidrug-resistant organisms. When such patients are placed on the waiting list for lung transplantation, the perioperative antibiotic regimen is usually planned ahead of time. In addition to results of bacterial cultures, any recent positive cultures for Aspergillus species or nontuberculous mycobacteria are considered when selecting the perioperative antimicrobial regimen.

For patients without previous airway cultures, prophylactic perioperative antibiotic therapy must provide coverage against multidrug-resistant gramnegative organisms and MRSA. At our center, patients are initiated on an antipseudomonal $\beta$-lactam (eg, cefepime [Maxipime], meropenem [Merrem] or piperacillin/tazobactam [Zosyn]) and vancomycin perioperatively. ${ }^{9}$ The choice of antipseudomonal agent should be based on the local sensitivities for each institution. This antibiotic combination is continued for 7 days unless an indication for a longer course of therapy arises in the postoperative period.

Surgical site infections in the postoperative period are most commonly caused by skin flora, the most frequent organism being Staphylococcus aureus. Patients colonized with gram-negative organisms preoperatively and those with surgical complications can develop pleural, and less commonly mediastinal, infectious complications in the postoperative period. Ischemic airway injury is the major risk factor for the development of Aspergillus tracheobronchitis. Rare occurrences of challenging surgical site infections have been observed in recipients with multidrug-resistant organisms present preoperatively. It seems logical, although largely not supported by evidence, that such patients should undergo longer and more vigorous infectious prophylaxis.

Fungal colonization and infections, most commonly Candida species or Aspergillus species, can occur in the perioperative period. Candida albicans remains the most common Candida species to be isolated from lung transplant recipients, although there has been a recent rise in non-albicans species. It is sometimes difficult to distinguish an invasive candidal infection from airway colonization. At our center, we usually treat patients with either fluconazole (Diflucan) or an echinocandin if they have ischemic airway injury or copious secretions and Candida species are the only organisms that are cultured. The major risk factors for Aspergillus infections are previous colonization and ischemic airway injury. ${ }^{10}$ Table 1 outlines some common prophylaxis regimens for viral, fungal, and Pneumocystis jiroveci infections.

\section{BACTERIAL INFECTIONS}

In contrast to nontransplant hosts, patients who have undergone lung transplantation are at much higher risk of becoming colonized and infected with drug-resistant organisms. Gram-negative organisms are the most frequent cause of bacterial pneumonia, with $P$ aeruginosa being the most common. Other gram-negative organisms identified less frequently include, but are not limited to, Acinetobacter baumannii, Escherichia coli, Klebsiella pneumoniae, Stenotrophomonas maltophilia, Burkholderia cepacia, and Serratia marcescens. De novo colonization of the airways with $P$ aeruginosa after transplantation has been demonstrated to be an independent risk factor for the development of BOS. ${ }^{6}$ Staphylococcus aureus is the most common gram-positive organism causing pneumonia and the second most common specific organism causing bacterial pneumonia among lung transplant recipients. ${ }^{10,11}$

Infection with $B$ cepacia has historically been associated with poor outcomes after transplantation, and many centers consider chronic infection with $B$ cepacia an absolute contraindication to lung transplantion. ${ }^{12,13}$ However, it has since been recognized that organisms formally classified as $B$ cepacia represent a group complex (B cepacia complex, BCC) comprising several distinct species (genomovars). Burkholderia cenocepacia (genomovar III) and Burkholderia multivorans (genomovar II) account for the majority of infections. ${ }^{14} \mathrm{~B}$ cenocepacia is associated with a much higher risk of 


\begin{tabular}{|c|c|c|}
\hline Infection & Viruses/Organisms & Common Prophylaxis Regimens \\
\hline Viral & $\begin{array}{l}\text { Herpes simplex virus } \\
\text { CMV }\end{array}$ & $\begin{array}{l}\text { Acyclovir, } 200 \mathrm{mg} \text {, po twice daily indefinitely } \\
\text { High risk }\left(D^{+} / \mathrm{R}^{-}\right) \\
\text {Valganciclovir, } 900 \mathrm{mg}, \text { po daily for } 6-12 \mathrm{mo} \\
\text { Medium Risk }\left(D^{+} / \mathrm{R}^{+}, \mathrm{D}^{-} / \mathrm{R}^{+}\right) \\
\text {1. Weekly CMV polymerase chain reaction, treat } \\
\text { when test result is positive } \\
\text { 2. Valganciclovir, } 900 \mathrm{mg} \text {, po daily for } 3-12 \mathrm{mo}\end{array}$ \\
\hline $\begin{array}{l}\text { Pneumocystis jiroveci } \\
\text { pneumonia }\end{array}$ & Pneumocystis jiroveci & $\begin{array}{l}\text { Trimethoprim/sulfamethoxazole, } 160 / 800 \mathrm{mg} \text {, } \\
\text { po } 3 \text { times weekly indefinitely } \\
\text { Alternatives for sulfa intolerant, all continued } \\
\text { indefinitely } \\
\text { 1. Atovaquone, } 1500 \mathrm{mg} \text {, po daily } \\
\text { 2. Pentamidine, } 300 \mathrm{mg} \text {, inhaled monthly } \\
\text { 3. Dapsone, } 100 \mathrm{mg} \text {, po daily }\end{array}$ \\
\hline Fungal & $\begin{array}{l}\text { Calbicans } \\
\text { Aspergillus species }\end{array}$ & $\begin{array}{l}\text { Until corticosteroid dose weaned postoperatively } \\
\text { or } 3 \text { mo } \\
\text { 1. Nystatin, } 5 \mathrm{~mL} \text {, po } 4 \text { times daily } \\
\text { 2. Fluconazole, } 100 \mathrm{mg} \text {, po daily } \\
\text { When colonized preoperatively or immediately } \\
\text { postoperatively, continued until cultures negative } \\
\text { 1. Voriconazole, } 200 \mathrm{mg} \text {, po twice daily } \\
\text { 2. Itraconazole, } 200 \mathrm{mg} \text {, po twice daily } \\
\text { 3. Amphotericin, } 20-40 \mathrm{mg} \text {, inhaled daily } \\
\end{array}$ \\
\hline
\end{tabular}

Abbreviation: po, by mouth (oral).

posttransplant mortality, including a 6-fold higher risk of death at 1 year compared with patients infected with $\mathrm{BCC}$ organisms other than $B$ cenocepacia and an 8-fold higher risk of death at 1 year compared with patients not infected with any BCC organism. ${ }^{15}$ In that series, BCC infection with organisms other than $B$ cenocepacia did not increase the risk of death after transplantation in comparison with patients not infected with BCC. $B$ cenocepacia can cause pneumonia, locally invasive disease with empyema, and disseminated infection. Although not a member of the BCC, Burkholderia gladioli has also been associated with an increased mortality. ${ }^{16}$ Transplant centers have to consider the specific species of Burkholderia and the risk of $B$ cenocepacia infection when evaluating patients with $\mathrm{CF}$ infected with these organisms.

Because lung transplant recipients are immunocompromised lifelong, empiric antibiotic treatment in lung recipients for pneumonia should include coverage for MRSA, $P$ aeruginosa, and atypical organisms (Listeria, Mycoplasma, Chlamydia). Based on the patient's clinical status, bronchoscopy with bronchoalveolar lavage, with or without transbronchial biopsies, should be considered. In addition to culture data, bronchoscopy helps to evaluate for noninfectious causes of shortness of breath and abnormal chest radiography, including airway complications and acute or chronic rejection. Lung transplant recipients often require up to 14 days of antibiotic therapy or longer if recovery is slow or airway cultures remain positive. As with any patient with pneumonia, the spectrum of the antibiotic therapy should be narrowed based on culture results when possible.

\section{VIRAL INFECTIONS CMV}

CMV infection is a significant cause of morbidity in all transplant recipients. Among lung recipients, CMV is the most common opportunistic infection. ${ }^{17}$ The major risk factor for the development of CMV disease is the interaction of the CMV status of the recipient and the CMV status of the donor. Patients who are CMV negative receiving CMVnegative donor organs $\left(\mathrm{D}^{-} / \mathrm{R}^{-}\right)$are at very low risk for CMV infection. CMV-positive recipients receiving either a CMV-positive or CMV-negative allograft $\left(D^{-} / R^{+}\right.$or $\left.D^{+} / R^{+}\right)$are at medium risk, and CMV-negative recipients receiving a CMVpositive allograft $\left(D^{+} / R^{-}\right)$are at the highest risk of developing CMV disease. CMV infection most commonly occurs within the first 3 to 6 months after 
transplantation, and the risk wanes after the first year in most recipients unless the immunosuppressive regimen is augmented. However, a minority of recipients have recurrent $\mathrm{CMV}$ infections beyond the first year after transplantation, and this can result in increased morbidity and mortality.

CMV prophylaxis may delay the onset of invasive disease. In general, there are 2 main prophylactic strategies, but practices in specific centers are highly variable. Most centers advocate valganciclovir (Valcyte) or intravenous ganciclovir (Cytovene) for 6 to 12 months in high-risk recipients $\left(D^{+} / R^{-}\right)$, and some encourage lifelong prophylaxis. In contrast, controversy exists on the optimal approach for recipients at medium risk $\left(\mathrm{D}^{-} / \mathrm{R}^{+}\right.$or $D^{+} / R^{+}$) for CMV disease. One strategy involves universal prophylaxis for all at-risk patients. The second strategy is based on monitoring and preemptive therapy at the time of identification of active viral replication, ideally before symptomatic infection. Using this strategy, medium-risk patients are monitored frequently (once to twice weekly), screening either for viral pp65 antigenemia or with a plasma polymerase chain reaction (PCR) for CMV. Assays vary, and different virology laboratory tests have different thresholds of positivity. At present, fewer centers rely on testing for pp65 antigenemia because the PCR assay has become more widely available and is less prone to technical errors. Treatment of CMV infection is started at the first sign of viral replication.

Concerning prophylactic therapy, most centers either start with intravenous ganciclovir and then transition to oral valganciclovir or administer oral valganciclovir from the outset. Valganciclovir is typically administered at a dose of $900 \mathrm{mg}$ orally daily for prophylaxis in patients with normal renal function, but the dose may need to be adjusted in the setting of low body mass or leukopenia. ${ }^{18}$ The most frequent adverse effects from ganciclovir or valganciclovir therapy are leukopenia and thrombocytopenia. There is also a small risk of ganciclovir resistance in patients on prophylaxis, especially if treatment is periodically interrupted. In addition, a limitation of prophylaxis for a given time course is shifting the timeline of CMV disease to a later time point after transplantation. CMV-specific immunoglobulin (Cytogam) is used at few centers as part of the prophylactic regimen for high-risk patients. ${ }^{19}$ No randomized controlled trials have demonstrated the superiority of universal prophylaxis over a preemptive strategy. However, one large multicenter trial demonstrated that in at-risk patients, 12 months of prophylaxis with valganciclovir was superior to 3 months after 13 months of follow-up with respect to CMV infection, with no change in acute rejection, CMV UL97 ganciclovir resistance mutations, or laboratory abnormalities. ${ }^{20}$

The term CMV infection applies to any situation in which viral replication is identified. CMV disease indicates CMV infection with symptoms, including mononucleosis-like syndrome with fever, malaise, and fatigue, or organ-specific invasion involving the lung (Fig. 1), liver, gastrointestinal tract, central nervous system, or retina. ${ }^{21}$ As discussed earlier, there is no universal standard of the viral load cutoff that represents a positive blood PCR result. However, if symptoms are present, the general consensus is to initiate treatment of CMV infection. Standard therapy includes intravenous ganciclovir, $5 \mathrm{mg} / \mathrm{kg}$ twice daily, or oral valganciclovir, $900 \mathrm{mg}$ twice daily, with dose adjustments for renal function and leukocyte count. Oral valganciclovir has been shown to be noninferior to intravenous ganciclovir in a mixed population of transplant recipients and can be considered as initial therapy, especially in patients with subclinical CMV infection and mild disease. ${ }^{22,23}$ Antiviral therapy is generally continued for at least 1 week after viral replication is no longer detectable. CMV-specific immunoglobulin can be
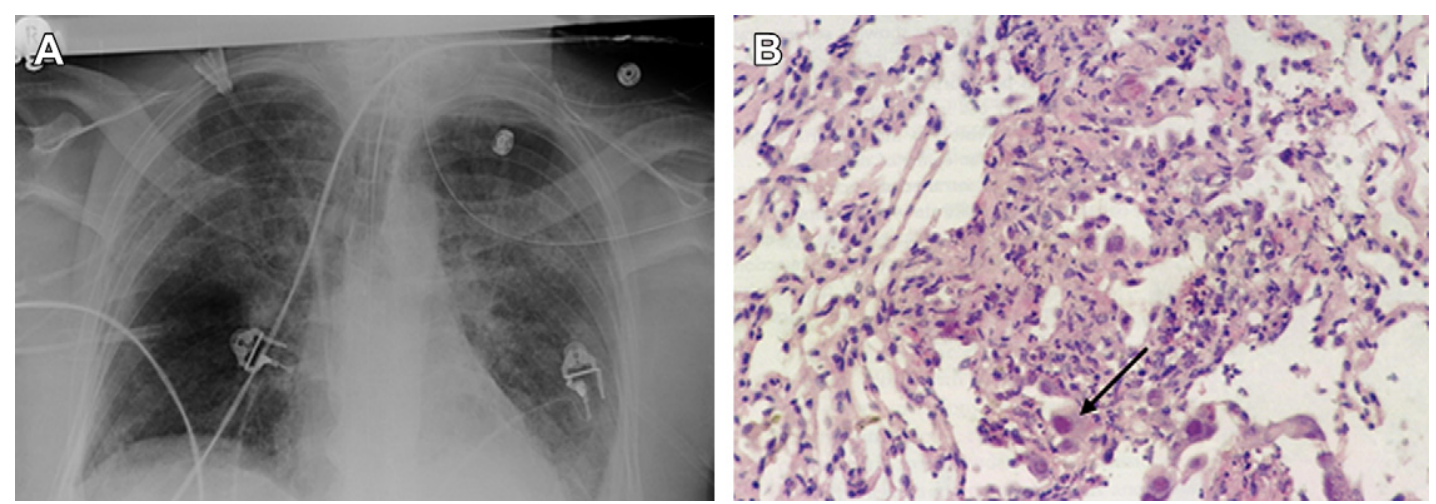

Fig. 1. (A) A bilateral lung recipient who CMV seropositive developed severe CMV pneumonia and respiratory failure. (B) Pathologic examination of CMV pneumonia with characteristic inclusion bodies (black arrow). 
used as add-on therapy in severe cases or in patients not responding to initial therapy, especially in seronegative recipients. In patients not responding to conventional therapy, resistance to ganciclovir must be evaluated. The UL 97 gene encodes a protein kinase involved in the phosphorylation and activation of ganciclovir, and mutations within this gene are the most common mechanisms of ganciclovir resistance. Mutations within the UL54 region, which encodes for CMV DNA polymerase, also confer ganciclovir resistance and are more likely to result in resistance to cidofovir and foscarnet. Many patients with UL 97 mutations, and some with UL54 mutations, respond to therapy with cidofovir or foscarnet; however, drug toxicity is the major obstacle to treatment.

\section{Community-Acquired Respiratory Viruses}

Community-acquired respiratory virus (CARV) infections, including respiratory syncytial virus (RSV), influenza, parainfluenza, rhinovirus, and adenovirus, have been associated with increased risk of BOS and death. ${ }^{24}$ Other CARVs have been identified, including human metapneumovirus, coronavirus, and bocavirus, but the role of these viruses in the development of BOS is not yet established.

Given that influenza infection is treatable, it is important to evaluate patients with clinical symptoms during the typical influenza season and treat them accordingly. As in nontransplant recipients, the first-line therapy for influenza in lung transplant recipients is the neuraminidase inhibitor oseltamivir (Tamiflu). When the severity of illness is high, the duration of therapy is often extended beyond the conventional 5 days to 7 to 14 days.

The decision to treat RSV with ribavirin, either inhaled or systemic, varies from center to center and between individual patients. The RSV season overlaps with the influenza season and may extend later into the spring. In patients with RSV, the goal is to reduce the progression from upper respiratory tract infection to lower respiratory tract infection and thus the risk of BOS. ${ }^{25}$ Given this risk, in addition to high clinical suspicion for influenza, patients with upper respiratory tract symptoms during the winter and early spring should be tested for RSV as well. At our center, patients with a positive result for nasopharyngeal viral swab for RSV are treated with 3 days of inhaled ribavirin therapy. Intravenous ribavirin therapy is limited by drug toxicity, especially hemolytic anemia. Other treatment modalities, including palivizumab, an immunomodulating RSV-specific monoclonal antibody; intravenous immunoglobulin (IVIG); and RSV-IVIG (no longer commercially available), have been evaluated in children and in hematopoietic stem cell transplant recipients. These agents seem to decrease viral loads in animal studies and have shown trends toward efficacy in clinical trials in the stem cell transplant population; however, few data exist in lung transplant recipients. ${ }^{26}$

\section{Epstein-Barr Virus and Posttransplant Lymphoproliferative Disorder}

The Epstein-Barr virus (EBV) is the agent that most commonly causes infectious mononucleosis. In transplant recipients, the major morbidity of EBV is posttransplant lymphoproliferative disorder (PTLD). There are 2 major subtypes of PTLD. Polymorphic PTLD is characterized by a monoclonal $B$-cell population in various stages of maturation and reactive T cells. Monomorphic PTLD is characterized by homogenous sheets of transformed monoclonal B cells, frequently with cytogenetic abnormalities. Monomorphic PTLD is a subtype of non-Hodgkin lymphoma. ${ }^{27}$

PTLD occurs in the setting of immunosuppression, and evidence of EBV infection has been identified in as many as $80 \%$ to $90 \%$ of patients with PTLD. ${ }^{28,29}$ PTLD frequently occurs in the first year after transplantation but can present years later. Among transplant recipients, those who have undergone heart and lung transplants are at the highest risk of developing PTLD, which is likely related to the higher intensity of immunosuppression maintained in these patients. ${ }^{30}$ The reported incidence of PTLD after lung transplantation varies between $2.5 \%$ and $8 \%$, with a recent series identifying 34 cases in 705 patients $(4.8 \%)$ who had undergone lung transplantation at a single center. ${ }^{31}$ PTLD frequently occurs in the transplanted organ, and, in lung recipients, this neoplasm can present as solitary or multiple pulmonary nodules or masses, hilar or mediastinal lymphadenopathy, or a pleural effusion. In general, cases of PTLD that develop in the first year after lung transplantation tend to be isolated to the chest, but cases that develop beyond the first year after transplantation tend to be extrathoracic, often involving the abdomen and pelvis. ${ }^{32,33}$ It is noteworthy that late extrathoracic cases tend to have a worse prognosis and are less likely to respond to de-escalation of immunosuppression.

The diagnosis of PTLD requires tissue examination, and, similar to the evaluation for lymphoma, excisional biopsies are ideal. Serum PCR testing for EBV has been evaluated as a tool to aid in the diagnosis and follow-up of patients with PTLD. While the specificity of a positive test in patients with PTLD is near $100 \%$, the sensitivity has been reported to be as low as $40 \%$ because many patients with pathologically confirmed PTLD will have negative serum EBV PCR results. ${ }^{34}$ 
The initial treatment of PTLD is generally a deescalation of immunosuppression. ${ }^{35}$ There is an increased risk of allograft rejection with this approach. The anti-CD20 monoclonal antibody rituximab (Rituxan) is the next line of therapy for PTLD. Rituximab is used when remission is not achieved with reduced immunosuppression. In addition, recent studies suggest that administering rituximab earlier in the course of PTLD improves the response and duration of remission. ${ }^{36,37}$ If reducing immunosuppression and administration of rituximab does not induce remission of PTLD, systemic chemotherapy with CHOP (cyclophosphamide, doxorubicin, vincristine, and prednisone) is usually administered. Surgical resection can be curative in cases of PTLD with localized disease. Radiation therapy can also be considered in cases of local diseases that are not amenable to surgical resection. When performed, both surgery and radiation should be combined with a reduction in immunosuppression with or without rituximab.

\section{FUNGAL INFECTIONS Prophylaxis}

Antifungal prophylaxis in the immediate posttransplant period varies widely from center to center. This variation is demonstrated in a survey of 50 lung transplant centers published in 2006. ${ }^{38}$ Universal prophylaxis and targeted prophylaxis (patients colonized with Aspergillus species before transplantation or becoming colonized after transplantation) were the 2 most common approaches in the survey. Regimens used by different centers included inhaled amphotericin B with or without an azole (usually itraconazole [Sporanox]), itraconazole alone, and fluconazole alone. Universal voriconazole (Vfend) prophylaxis has been shown to decrease the incidence of invasive aspergillosis at 1 year in comparison with targeted prophylaxis with itraconazole with or without inhaled amphotericin B; however, there were increased adverse events in the treatment group, predominantly an elevation of serum liver enzyme levels suggestive of hepatic toxicity. ${ }^{39}$ At our center, we practice a targeted approach using itraconazole or voriconazole in patients colonized with Aspergillus before transplantation or immediately after transplantation. In addition, patients are administered prophylaxis with nystatin or fluconazole for oropharyngeal thrush until the corticosteroid doses have been tapered.

\section{Aspergillus Species Infections}

Aspergillus fumigatus causes most fungal infections in lung transplant recipients. However, other species, including Aspergillus flavus, Aspergillus niger, Aspergillus terreus, and Aspergillus ustus, have been increasingly reported as causes of invasive fungal infection. ${ }^{40}$ Aspergillus infections can be classified as localized airway infection or invasive disease. Invasive diseases include Aspergillus tracheobronchitis, invasive pulmonary aspergillosis, and disseminated aspergillosis. ${ }^{41}$

Aspergillus tracheobronchitis is characterized by the involvement of anastomotic sites and distal airways, especially areas of ischemic injury without extension into the lung parenchyma. Necrosis, ulceration, and pseudomembrane formation are the characteristic features of Aspergillus tracheobronchitis, and the diagnosis is made using bronchoscopy. ${ }^{42}$ The risk of developing Aspergillus tracheobronchitis is highest in the first 3 months after transplantation. Progression of the infection is possible if treatment is delayed.

In contrast to the stem cell transplant population, patients who have undergone any solid organ transplant and develop invasive pulmonary aspergillosis do not regularly demonstrate the characteristic halo sign radiologic finding on computed tomography of the chest. ${ }^{43,44}$ The most common radiologic findings are nonspecific, including focal or multifocal consolidation, infiltration, or nodular lesions with or without cavitation (Fig. 2).

The diagnosis of invasive Aspergillus infections is based on appropriate clinical signs and symptoms combined with pathologic and microbiological confirmation. The Aspergillus galactomannan antigen assay has been used as a marker of angioinvasive disease, especially in the stem cell recipient population, but the sensitivity of the assay for the diagnosis of invasive disease in lung transplant recipients has been reported to be only $30 \%$ and is especially poor in patients with

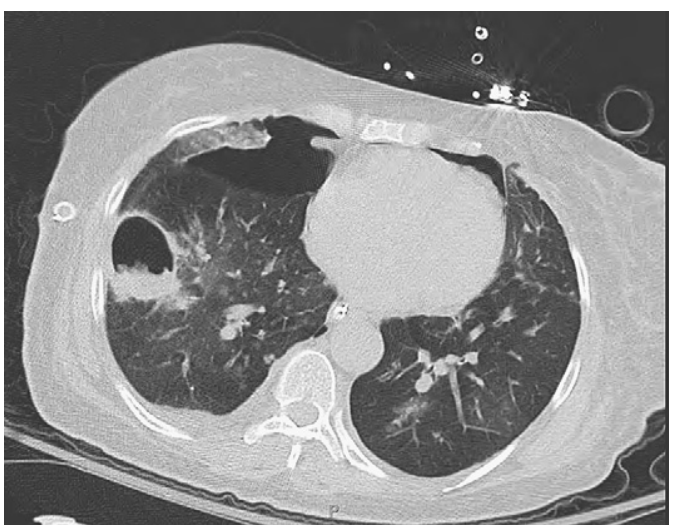

Fig. 2. A bilateral lung transplant recipient developed a thick-walled right lower lobe cavity, which was confirmed to be due to invasive aspergillosis histologically. The recipient developed respiratory failure and pneumothorax and did not respond to therapy. 
tracheobronchitis. ${ }^{45}$ Testing the bronchoalveolar fluid for the presence of the galactomannan antigen appears to be more sensitive than testing the serum when looking for tracheobronchitis or invasive pulmonary aspergillosis; however, the overall clinical condition of the patient must be considered before deciding on any one diagnostic test to make the diagnosis of invasive aspergillosis. ${ }^{46}$ False-positive results of the serum galactomannan assay can occur in the presence of certain antibiotics, especially piperacillin/tazobactam.

The treatment of Aspergillus infections involves the use of azoles, echinocandins, and amphotericin $B$ and generally depends on the severity of illness, with the lipid formulation of amphotericin B remaining the first line of treatment in severe disease. There is emerging data that echinocandins, (caspofungin [Cancidas], micafungin [Mycamine], and anidulafungin [Eraxis]), alone or in combination with voriconazole may be an effective first-line treatment of pulmonary aspergillosis, with significantly less toxicity. ${ }^{47,48}$ Voriconazole is generally preferred over itraconazole, given the more predicable bioavailability of voriconazole over itraconazole. Posaconazole (Noxafil) also has activity against Aspergillus species, but there is not as much experience with this agent, and it is not available in an intravenous formulation. In addition, azoles have a potent interaction with calcineurin inhibitors, and the cyclosporine (Neoral) or tacrolimus (Prograf) doses need to be significantly reduced when itraconazole or voriconazole are inititated. ${ }^{41}$

\section{Candida Species Infections}

$C$ albicans remains the most common Candida species causing fungal infections in lung transplant recipients but a shift toward non-albicans species has been observed. ${ }^{10}$ Clinical patterns of candidiasis in lung transplantation recipients range from mucocutaneous to invasive disease, with candidemia and multiorgan involvement. Although $C$ albicans is generally sensitive to azole agents, including fluconazole, some non-albicans Candida species are resistant to fluconazole. Several recent studies have demonstrated the effectiveness of echinocandins in invasive candidiasis and should be considered as a first-line treatment pending identification and speciation of the Candida species in culture. ${ }^{49-51}$ Amphotericin B is effective for invasive candidiasis; however, given its toxicity and the effectiveness of safer echinocandins and azoles, it should be reserved for treatment failures or patients who are intolerant of echinocandins and are infected by organisms that are resistant to azole agents.

\section{P jiroveci}

$P$ jiroveci (formerly Pneumocystis carinii) is an organism that is ubiquitous in the environment and can cause pneumonia in any immunosuppressed patient. In the era of prophylaxis, it is rare for patients to develop $P$ jiroveci pneumonia. ${ }^{52}$ The first-line prophylactic is trimethoprim/sulfamethoxazole (Bactrim, Septra), although in patients who are allergic to sulfa drugs alternative options include inhaled pentamidine (Pentam), atovaquone (Mepron), and dapsone. This infection is treated with high doses of trimethoprim/sulfamethoxazole, often with an increase in the corticosteroid dose, especially when complicated by hypoxemia. ${ }^{53}$ In patients who are allergic to sulfa drugs, desensitization can be considered in order to use trimethoprim/sulfamethoxazole for either prophylaxis or treatment in the event of Pneumocystis pneumonia.

\section{Other Fungi}

Many other fungal pathogens have been associated with clinical disease in lung transplant recipients. Cryptococcus neoformans can infect the lung itself, in addition to causing disseminated multisystem disease. Depending on the geography, endemic fungi can cause disease in lung transplant recipients (eg, histoplasmosis, coccidioidomycosis, and blastomycosis). In addition, several non-Aspergillus mycelial fungi, including zygomycetes (eg, mucormycosis) and Scedosporium species, have more recently been recognized as important pathogens in the transplant recipient population and are generally associated with worsened outcomes compared with Aspergillus species. ${ }^{54}$

\section{MYCOBACTERIA \\ Nontuberculosis Mycobacteria}

Nontuberculosis mycobacterial infection before lung transplantation is generally observed in patients with bronchiectasis, especially CF. The prevalence of these organisms has been reported as $3 \%$ to $10 \%$ in patients with adult onset bronchiectasis and $13 \%$ to $28 \%$ in patients with CF. ${ }^{55,56}$ The risk of recurrence of nontuberculosis mycobacterial infection and significant clinical disease seems to be the highest with Mycobacterium abscessus, although in one study the posttransplant course was not affected by $M$ abscessus infection. ${ }^{57}$ Although difficult to manage, it is possible to successfully treat infections caused by Mycobacterium avium-intracellulare (Fig. 3) and $M$ abscessus in lung transplant recipients. ${ }^{58}$ Treatment is guided by the American Thoracic Society (ATS)/Infectious Diseases Society of America (IDSA) statement regarding nontuberculous mycobacterial disease 


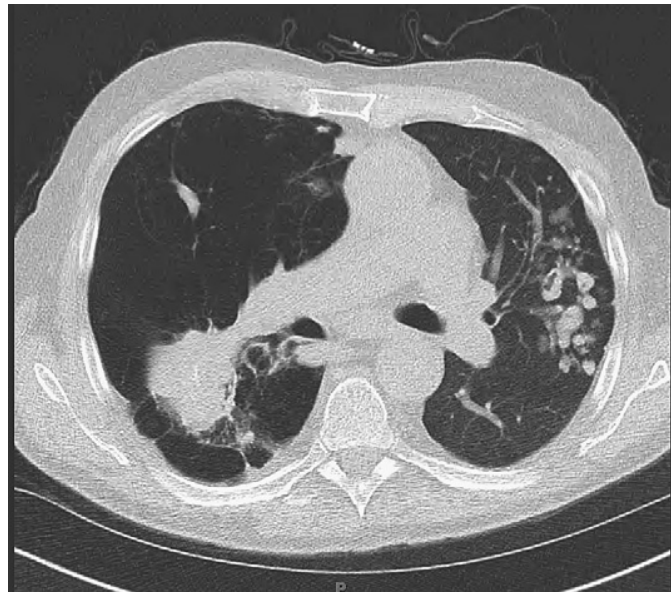

Fig. 3. A left single lung transplant recipient developed a masslike infiltrate in the native emphysematous lung and multiple nodules in the allograft. Pathologic conditions were consistent with granulomatous inflammation and cultures showed positive results for Mycobacterium avium-intracellulare. The recipient responded well to treatment.

and is the same as in the nontransplant immunocompetent host. ${ }^{59}$

\section{Mycobacterium tuberculosis}

Transplant recipients should be evaluated for latent infection with Mycobacterium tuberculosis using the tuberculin skin test. In the event of a positive test result and no evidence of active tuberculosis on chest radiograph, INH therapy should be administered for 6 to 9 months. ${ }^{60}$ Active tuberculosis should be treated according to ATS/Centers for Disease Control/IDSA guidelines and is the same as in the nontransplant host. ${ }^{61}$

\section{SUMMARY}

Throughout their lives lung transplant recipients are at an increased risk of infectious complications from the immediate postoperative period. Appropriate antimicrobial prophylaxis, knowledge of the timing of risk of infectious complications, and aggressive appropriate treatment of the infections decrease morbidity and mortality in this population. Many infectious complications, including bacterial, viral, and fungal infections, confer an increased risk for BOS, the leading cause of death in lung recipients after more than 1 year after transplantation. Continued investigations regarding the ideal antimicrobial prophylaxis and immunosuppressive regimens will allow clinicians caring for lung transplant recipients to minimize the risks of infection, maximize allograft function by limiting rejection, and minimize the adverse effects that are associated with prolonged antimicrobial therapy.

\section{REFERENCES}

1. Christie JD, Edwards LB, Kucheryavaya AY, et al. The registry of the International Society for Heart and Lung Transplantation: twenty-eighth adult lung and heart-lung transplant report-2011. J Heart Lung Transplant 2011;30(10):1104-22.

2. Alexander BD, Tapson VF. Infectious complications of lung transplantation. Transpl Infect Dis 2001;3(3): 128-37.

3. Snyder LD, Finlen-Copeland A, Jackson W, et al. Cytomegalovirus pneumonitis is a risk for bronchiolitis obliterans syndrome in lung transplantation. Am J Respir Crit Care Med 2010;181:1391-6.

4. Vos R, Vanaudenaerde BM, Geudens N, et al. Pseudomonal airway colonization: risk factor for bronchiolitis obliterans syndrome after lung transplantation? Eur Respir J 2008;31:1037-45.

5. Gottlieb J, Mattner F, Weissbrodt H, et al. Impact of graft colonization with gram-negative bacteria after lung transplantation on the development of bronchiolitis obliterans syndrome in recipients with cystic fibrosis. Respir Med 2009;103:743-9.

6. Botha P, Archer L, Anderson RL, et al. Pseudomonas aeruginosa colonization of the allograft after lung transplantation and the risk of bronchiolitis obliterans syndrome. Transplantation 2008;85:771-4.

7. Weigt SS, Elashoff RM, Huang C, et al. Aspergillus colonization of the lung allograft is a risk factor for bronchiolitis obliterans syndrome. Am J Transplant 2009;9:1903-11.

8. Campos S, Caramori M, Teizeira R, et al. Bacterial and fungal pneumonias after lung transplantation. Transplant Proc 2008;27:528-35.

9. Chang ST, Krupnick AS. Perioperative antibiotics in thoracic surgery. Thorac Surg Clin 2012;22:35-45.

10. Solé A, Salavert M. Fungal infections after lung transplantation. Curr Opin Pulm Med 2009;15: 243-53.

11. Aguilar-Guisado M, Givalda J, Ussetti P, et al. Pneumonia after lung transplantation in the RESITRA Cohort: a multicenter prospective study. Am J Transplant 2007;7:1989-96.

12. Chaparro C, Maurer J, Gutierrez C, et al. Infection with Burkholderia cepacia in cystic fibrosis: outcome following lung transplantation. Am J Respir Crit Care Med 2001;163:43-8.

13. Meachery G, De Soyza A, Nicholson A, et al. Outcomes of lung transplantation for cystic fibrosis in a large UK cohort. Thorax 2008;63:725-31.

14. Luong M, Morrissey O, Husain S. Assessment of infection risks prior to lung transplantation. Curr Opin Infect Dis 2010;23:578-83. 
15. Alexander BD, Petzold EW, Reller LB, et al. Survival after lung transplantation of cystic fibrosis patients infected with Burkholderia cepacia complex. Am J Transplant 2008;8:1025-30.

16. Murray S, Charbeneau J, Marshall BC, et al. Impact of Burkholderia infection on lung transplantation in cystic fibrosis. Am J Respir Crit Care Med 2008; 178:363-71.

17. Zamora MR. Cytomegalovirus and lung transplantation. Am J Transplant 2004;4:1219-26.

18. Kotton CN, Kumar D, Caliendo AM, et al. International consensus guidelines on the management of cytomegalovirus in solid organ transplantation. Transplantation 2010;89:779-95.

19. Zuk DM, Humar A, Weinkauf JG, et al. An international survey of cytomegalovirus management practices in lung transplantation. Transplantation 2010; 90:672-6.

20. Palmer SM, Limaye AP, Banks M, et al. Extended valganciclovir prophylaxis to prevent cytomegalovirus after lung transplantation. Ann Intern Med 2010;152:761-9.

21. Kotloff RM, Ahya VN. Medical complications of lung transplantation. Eur Respir J 2004;23:334-42.

22. Asberg A, Humar A, Rollag H, et al. Oral valganciclovir is noninferior to intravenous ganciclovir for the treatment of cytomegalovirus disease in solid organ transplant recipients. Am J Transplant 2007;7:2106-13.

23. Snydman DR, Limaye AP, Potena L, et al. Update and review: state-of-the-art management of cytomegalovirus infection and disease following thoracic organ transplantation. Transplant Proc 2011;43:S1-17.

24. Khalifah AP, Hachem RR, Chakinala MM, et al. Respiratory viral infections are a distinct risk for bronchiolitis obliterans syndrome and death. Am J Respir Crit Care Med 2004;170:181-7.

25. Chakinala MM, Walter MJ. Community acquired respiratory viral infections after lung transplantation: clinical features and long-term consequences. Semin Thorac Cardiovasc Surg 2004;16:342-9.

26. Shah JN, Chemaly RF. Management of RSV infections in adult recipients of hematopoietic stem cell transplantation. Blood 2011;117:2756-63.

27. Harris NL, Ferry JA, Swerdlow SH. Posttransplant lymphoproliferative disorders: summary of society for hematopathology workshop. Semin Diagn Pathol 1997;14:8-14.

28. Armitage JM, Kormos RL, Stuart RS, et al. Posttransplant lymphoproliferative disease in thoracic organ transplant patients: ten years of cyclosporinebased immunosuppression. J Heart Lung Transplant 1991;10:877-86.

29. Nalesnik MA. Posttransplantation lymphoproliferative disorders (PTLD): current perspectives. Semin Thorac Cardiovasc Surg 1996;8:139-48.

30. Loren AW, Tsai DE. Post-transplant lymphoproliferative disorder. Clin Chest Med 2005;26:631-45.
31. Kremer BE, Reshef R, Misleh JG, et al. Post-transplant lymphoproliferative disorder after lung transplantation: a review of 35 cases. J Heart Lung Transplant 2012;31(3):296-304.

32. Paranjothi S, Yusen R, Kraus M, et al. Lymphoproliferative disease after lung transplantation: comparison of presentation and outcome of early and late cases. J Heart Lung Transplant 2001;20:1054-63.

33. Hachem RR, Chakinala MM, Yusen RD, et al. Abdominal-pelvic Iymphoproliferative disease after lung transplantation: presentation and outcome. Transplantation 2004;77:431-7.

34. Tsai DE, Nearey M, Hardy CL, et al. Use of EBV PCR for the diagnosis and monitoring of post-transplant lymphoproliferative disorder in adult solid organ transplant patients. Am J Transplant 2002;2:946-54.

35. Jagadeesh D, Woda BA, Draper J, et al. Post transplant lymphoproliferative disorders: risk, classification, and therapeutic recommendations. Curr Treat Options Oncol 2012;13(1):122-36.

36. Evens AM, David KA, Helenowski I, et al. Multicenter analysis of 80 solid organ transplantation recipients with post-transplantation lymphoproliferative disease: outcomes and prognostic factors in the modern era. J Clin Oncol 2010;28:1038-46.

37. Trappe R, Choquet S, Oertel SH, et al. Sequential treatment with rituximab followed by $\mathrm{CHOP}$ chemotherapy in adult B-cell post-transplant lymphoproliferative disorder (PTLD): the prospective international multicentre phase 2 PTLD-1 trial. Lancet Oncol 2012;13(2):196-206.

38. Husain S, Zaldonis D, Kusne S, et al. Variation in antifungal prophylaxis strategies in lung transplantation. Transpl Infect Dis 2006;8:213-8.

39. Husain S, Paterson DL, Studer S. Voriconazole prophylaxis in lung transplant recipients. Am J Transplant 2006;6:3008-16.

40. Caston JJ, Linares MJ, Gallego C, et al. Risk factors for pulmonary Aspergillus terreus infection in patients with positive culture for filamentous fungi. Chest 2007;131:230-8.

41. Silveira PF, Husain S. Fungal infections in lung transplant recipients. Curr Opin Pulm Med 2008; 14:211-8.

42. Kramer MR, Denning DW, Marshall SE, et al. Ulcerative tracheobronchitis after lung transplantation: a new form of invasive aspergillosis. Am Rev Respir Dis 1991;144:552-6.

43. Singh N, Husain S. Aspergillus infections after lung transplantation: clinical differences in type of transplant and implications for management. J Heart Lung Transplant 2003;22:258-66.

44. Paterson DL, Singh N. Invasive aspergillosis in transplant recipients. Medicine 1999;78:123-38.

45. Husain S, Kwak EJ, Obman A, et al. Prospective assessment of Platelia Aspergillus galactomannan antigen for the diagnosis of invasive aspergillosis in 
lung transplant recipients. Am J Transplant 2004;4: 796-802.

46. Husain S, Paterson DL, Studer SM, et al. Aspergillus galactomannan antigen in the bronchoalveolar lavage fluid for the diagnosis of invasive aspergillosis in lung transplant recipients. Transplantation 2007;83:1330-6.

47. Groetzner J, Kaczmarek I, Wittwer T, et al. Caspofungin as first-line therapy for the treatment of invasive aspergillosis after thoracic organ transplantation. J Heart Lung Transplant 2008;27:1-6.

48. Singh N, Limaye AP, Forrest $\mathrm{G}$, et al. Combination of voriconazole and caspofungin as primary therapy for invasive aspergillosis in solid organ transplant recipients: a prospective, multicenter, observational study. Transplantation 2006;81:320-6.

49. Mora-Duarte J, Betts R, Rotstein C, et al. Comparison of caspofungin and amphotericin $\mathrm{B}$ for invasive candidiasis. N Engl J Med 2002;347:2020-9.

50. Kuse ER, Chetchotisakd $P$, da Cunha CA, et al. Micafungin versus liposomal amphotericin B for candidaemia and invasive candidosis: a phase II randomized double-blind trial. Lancet 2007;369: 1519-27.

51. Reboli AC, Rotstein C, Pappas PG, et al. Anidulafungin versus fluconazole for invasive candidiasis. N Engl J Med 2007;356:2472-82.

52. Green H, Paul M, Vidal L, et al. Prophylaxis of Pneumocystis pneumonia in immunocompromised non-HIV-infected patients: systematic review and meta-analysis of randomized controlled trials. Mayo Clin Proc 2008;82:1052-9.

53. Briel M, Bucher HC, Boscacci R, et al. Adjunctive corticosteroids for Pneumocystis jiroveci pneumonia in patients with HIV-infection. Cochrane Database Syst Rev 2006;3:CD006150.

54. Husain S, Alexander B, Munoz P, et al. Opportunistic mycelia fungal infections in organ transplant recipients: emerging importance of non-Aspergillus mycelia fungi. Clin Infect Dis 2003;37:221-9.

55. Fowler SJ, French J, Screaton NJ, et al. Nontuberculous mycobacteria in bronchiectasis: prevalence and patient characteristics. Eur Respir J 2006;28: 1204-10.

56. Lipuma JJ. The changing microbial epidemiology in cystic fibrosis. Clin Microbiol Rev 2010;(23):299-323.

57. Chalermskulrat W, Sood N, Neuringer IP, et al. Nontuberculous mycobacteria in end stage cystic fibrosis: implications for lung transplantation. Thorax 2006;61:507-13.

58. Chernenko SM, Humar A, Hutcheon M, et al. Mycobacterium abscessus infections in lung transplant recipients: the international experience. J Heart Lung Transplant 2006;25:1447-55.

59. Griffith DE, Aksamit T, Brown-Elliot BA, et al. An official ATS/IDSA statement: diagnosis, treatment, and prevention of nontuberculous mycobacterial disease. Am J Respir Crit Care Med 2007;175:367-416.

60. Aguado JM, Torre-Cisneros J, Fortun J, et al. Tuberculosis in solid-organ transplant recipients: consensus statement of the group for the study of infection in transplant recipients (GESITRA) of the Spanish Society of Infectious Diseases and Clinical Microbiology. Clin Infect Dis 2009;48:1276-84.

61. Centers for Disease Control, Prevention. Treatment of tuberculosis. American Thoracic Society, CDC, and Infectious Diseases Society of America. MMWR 2003;52(RR-11):1-72. 